\title{
Decreased MiR-200a/141 Suppress Cell Migration and Proliferation by Targeting PTEN in Hirschsprung's Disease
}

\author{
Hongxing Lijacc Junwei Tang ${ }^{a, c}$ Hao Lei ${ }^{a, c}$ Peng Caia ${ }^{a, c}$ Hairong Zhu ${ }^{a, c}$ Bo Lia,c \\ Xiaoqun Xu $\mathrm{u}^{\mathrm{a}, \mathrm{c}}$ Yankai Xia ${ }^{\mathrm{a}, \mathrm{b}}$ Weibing Tang ${ }^{\mathrm{a}, \mathrm{c}}$ \\ aState Key Laboratory of Reproductive Medicine, Institute of Toxicology, School of Public Health, \\ Nanjing Medical University, ${ }^{b}$ Key Laboratory of Modern Toxicology (Nanjing Medical University), \\ Ministry of Education, cDepartment of Pediatric Surgery, Nanjing Children's Hospital Affiliated Nanjing \\ Medical University, Nanjing, China
}

\section{Key Words}

HSCR • 3'-UTR • Neural crest cell • Neural development • Pediatric

\begin{abstract}
Background/Aims: Hirschsprung's disease (HSCR) is a genetic disorder of neural crest development. In this study, we investigated whether and how miR-200a and miR-141, belonging to miR-200 family, were involved in the pathogenesis of HSCR. Methods: Quantitative real time PCR and Western blot were used to detect the levels of miRNA, mRNAs, and proteins in colon tissues from $88 \mathrm{HSCR}$ patients and 75 controls. The direct regulation of specific mRNA by miRNAs was validated by dual-luciferase reporter assay and RNA interference in cell lines. Transwell assays, CCK8 assay, and flow cytometry were inplemented to measure viability and activities of human 293T and SH-SY5Y cells, respectively. Results: Aberrant suppression of miR-200a was observed in colon tissues of HSCR patients. A decreased level of miR-200a and miR-141 correlated with increased levels of PTEN mRNA and protein. The Dual-Luciferase reporter gene assay demonstrated that miR-200a and miR-141 binded directly to 3'UTR of PTEN and resulting in the inhibition of PTEN. The reductions in miR-200a and miR-141 inhibited migration and proliferation of $293 \mathrm{~T}$ and SH-SY5Y cells through up-regulating the expression of PTEN. Moreover, knocking-down of PTEN rescued the extent of suppressed cell migration and proliferation induced by miR-200a and miR-141. Conclusions: The miR-200 family may play a crucial role in the pathogenesis of HSCR by co-regulating PTEN.
\end{abstract}




\section{Cellular Physiology and Biochemistry}

Cell Physiol Biochem 2014;34:543-553

\begin{tabular}{l|l}
\hline DOI: $10.1159 / 000363021$ & (C) 2014 S. Karger AG, Basel
\end{tabular}

\begin{tabular}{l|l} 
Published online: August 08, 2014 & www.karger.com/cpb
\end{tabular}

Li et al.: MiR-200a/141 in Hirschsprung's Disease

\section{Introduction}

Hirschsprung's disease (HSCR), as a rare alimentary tract disease, is caused by the absence of ganglion cells in the submucosal and myenteric plexi of the gut [1]. HSCR occurs in about 1:2000-1:5000 live births with sex ratio bias that males is four times more likely to be affected than females [2]. It features that the enteric neural crest cells (ENCCs) stop migrating, leading to the failure of reaching to the hindgut during embryogenesis from 5 th to 12 th weeks that results in aganglionosis of the distal gut. It is highly associated with the ENCCs migration, proliferation, differentiation, survival, and/or apoptosis [3]. The high recurrence among siblings and the occurrence of HSCR as part of the phenotype of various syndromes imply the importance of genetic factors [4]. Extensive research has identified a number of key genes that regulate neural crest cell development in the pathogenesis of HSCR including RET, GDNF, GFRa1, NRTN, EDNRB, ET3, ZFHX1B, PHOX2b, SOX10, and SHH [5]. However, few researches have been reported on the development associated non-coding genes, such as miRNAs, which may offer a novel approach to explore new pathways that contribute to HSCR.

MicroRNAs are an abundant class of short, non-coding RNA molecules that mediate the regulation of target genes post-transcriptionally through complementary binding to the 3'-untranslated regions (3'-UTR) of target mRNAs and that have emerged as master regulators in diverse physiologic and pathologic processes [6]. Recently, a set of miRNAs play crucial roles in disease occurrence through the common target genes by regulating cell differentiation, proliferation, migration and apoptosis were reported in many studies, the data shown that miR-200 family played important roles in disease occurrence [7-10]. MiR141 , as a part of the miR-200 family, shows responsibilities on the pathogenesis of HSCR by the suppressed cell migration and proliferation, which has been reported in our previous study [11]. However, the role of another member of the miR-200 family, miR-200a, which shares the common seed sequence with the miR-141, is still poorly understood, especially the potential common target gene which may be involved in HSCR progression.

In this study, we investigated the functional involvement of miR-200a and miR-141 in HSCR progression, based on the interpretations that the miR-200 family is closely related with cell migration and proliferation abilities $[10,12]$. We also identified the common targets of the two miRNAs, the phosphatase and tensin homolog (PTEN), a phosphatase which is critical for regulating the activation of the PI3K/PTEN/Akt/mTOR pathway involved in cell migration and proliferation [13]. In this study, we conducted the experiment to explore the mechanism of the miRNAs-target interactions.

\section{Materials and Methods}

Ethics statement and patient tissue samples

88 patients with a pathological diagnosis of HSCR and 75 matched controls colon tissues were collected from patients who underwent enterectomy in Nanjing Children's Hospital Affiliated to Nanjing Medical University from October 2009 to May 2013 (NJMU Birth Cohort). All the HSCR patients were diagnosed by barium enema and anorectal manometry evaluation before surgical procedures and pathological analysis for definite diagnosis after surgery. The matched controls, admitted to hospital for surgery because of intussusception or incarcerated and strangulated inguinal hernia without the ischemia or necrosis parts, were proven to be without HSCR or other congenital malformations. All samples were collected with the informed consent of the patients and were immediately frozen and stored at $-80^{\circ} \mathrm{C}$. The study was approved by the Institutional Ethics Committee of Nanjing Medical University. Additionally, all of studies were done under compliance with the government policies and the Helsinki Declaration.

RNA isolation and quantitative real-time RT-PCR ( $q R T-P C R)$

Total RNA, containing miRNA, was extracted from tissue specimens and cell lines by using Trizol reagent (Life Technologies, CA, USA) according to the manufacturer's instructions. TaqMan® MicroRNA 


\section{Cellular Physiology and Biochemistry}

Cell Physiol Biochem 2014;34:543-553

Li et al.: MiR-200a/141 in Hirschsprung's Disease

Assays (Applied Biosystems, CA, USA) was used as the probe for has-miR-200a, has-miR-141 and human U6 RNA which was amplified as an internal control. For the detection of mRNA, human GAPDH RNA was used as a control. The miRNA or mRNA levels were calculated according to $2^{-\Delta \Delta C t}$. Forward (F) and reverse (R) primer sequences were as follows: PTEN (F) 5'-TGGATTCGACTTAGACTTGACCT-3' and 5'-GGTGGGTTATGGTCTTCAAAAGG-3'; GAPDH (F) 5'-GCACCGTCAAGGCTGAGAAC-3' and (R) 5'-GGATCTCGCTCCTGGAAGATG-3'.

\section{Protein extraction and Western blotting}

The tissues and cell lysates were prepared by using a RIPA buffer (cOmplete, ULTRA, Mini, EDTAfree, EASYpack, Roche, Basel, Switzerland). The membranes with the target protein were incubated with the Primary antibody against PTEN (cat. \#7912-1, Epitomics, Burlingame, CA, USA) at $4{ }^{\circ} \mathrm{C}$ overnight and further incubated with the secondary antibodies, an anti-rabbit HRP-linked which was purchased from Beyotime (Nantong, Jiangsu, China) for $1 \mathrm{~h}$ at room temperature. The blots were developed using ECL reagent (Millpore, MASS, USA). Equal amount of protein loading in each lane was confirmed using GAPDH antibody. ImageJ software was used to quantify Western blot data.

\section{Dual-luciferase reporter assay}

For luciferase reporter experiments, the wild-type and mutated 3'-UTR sequence of PTEN mRNA were inserted into the KpnI and SacI sites of pGL3 promoter vector (Genscript, Nanjing, China), which were named pGL3-PTEN and pGL3-PTEN-mut, respectively. Cells were plated onto 24 -well plates at $2 \times 10^{9}$ cells/well and transfected with $100 \mathrm{ng}$ of pGL3-PTEN, pGL3-PTEN-mut, and $50 \mathrm{nM}$ miR-200a and miR-141 mimics and negative control, respectively. Firefly and Renilla luciferase activities were measured consecutively by using the Dual Luciferase Assay (Promega, Madison, WI) after $48 \mathrm{~h}$ transfection according to the manufacturer's protocols. Transfection was repeated three times in triplicate.

\section{Cell culture and transfection}

Human 293T cell and SH-SY5Y cell were obtained from American Type Culture Collection (ATCC, Manassas VA, USA), which were cultured in complete growth medium DMEM (Hyclone, UT, USA) supplemented with $10 \%$ heat-inactivated fetal bovine serum (10\% FBS), penicillin $(100 \mathrm{U} / \mathrm{ml})$, and streptomycin $(100 \mu \mathrm{g} / \mathrm{mL})$ at $37^{\circ} \mathrm{C}, 5 \% \mathrm{CO} 2$. The mimics and inhibitor of miR-200a, miR-141 and negative controls (GenePharma, Shanghai, China) were used in transfection experiments with Lipofectamine 2000 Reagent (Invitrogen, CA, USA) following the manufacturer's instructions.

\section{Cell transwell assays}

Cell transwell assays was evaluated using Transwell migration chambers $(8 \mu \mathrm{m}$ pore size, Millipore Corporation, Billerica, MA). 100 ul cell suspension with serum-free medium were seeded in the upper chamber $(1 \times 106$ cells $/ \mathrm{ml})$. The lower chamber contained $600 \mu$ l medium with $10 \%$ FBS as chemotaxin. After $48 \mathrm{~h}$ transfection, cells were stained with crystal violet staining solution (Beyotime, Nantong, China). Migrated cells were counted using Image-pro Plus 6.0 while cell numbers of normal control group were normalized to 1 . All experiments were performed in triplicate independently.

\section{Cell proliferation assays}

CCK-8 assay (Beyotime, Nantong, China) was used to detect the cell proliferation after $24 \mathrm{~h}$ transfection, accompanied with the employment of the TECAN infinite M200 Multimode microplate reader (Tecan, Mechelen, Belgium)to measure the absorbance at $450 \mathrm{~nm}$. All experiments were performed in triplicate independently.

\section{Cell cycle and apoptosis analysis}

For the cell cycle assay, at $48 \mathrm{~h}$ post-transfection, cells were collected and detected by BD Biasciences FACS Calibur Flow Cytometry (BD Biasciences, NJ, USA). For cell apoptosis analysis, cells were harvested and stained with the Annexin V-FITC/Propidium Iodide Kit (KeyGen Biotech, Nanjing, China) according to the manufacturer's instructions. Data were analyzed with FlowJo V7 software (Tree Star, Oregon, USA). All experiments were performed in triplicate independently. 


\section{Cellular Physiology and Biochemistry}

Cell Physiol Biochem 2014;34:543-553

\begin{tabular}{l|l}
\hline DOI: $10.1159 / 000363021$ & (c) 2014 S. Karger AG, Basel
\end{tabular}

Published onIIne: August 08, 2014

Li et al.: MiR-200a/141 in Hirschsprung's Disease

Table 1. Demographic and clinical features of study subjets, ${ }^{a}$ Student t-test, ${ }^{b}$ Two-sided $x^{2}$ test

\begin{tabular}{lccc}
\hline \multicolumn{1}{c}{ Variable } & $\begin{array}{c}\text { HSCR } \\
\mathrm{n}=88\end{array}$ & $\begin{array}{c}\text { Control } \\
\mathrm{n}=75\end{array}$ & $P$ \\
\hline Age, (months, mean, SE) & $3.90(0.23)$ & $3.17(0.26)$ & $0.26^{a}$ \\
Sex (\%) & & & \\
Male & $72(81.8)$ & $56(74.6)$ & $0.27^{b}$ \\
Female & $16(18.1)$ & $19(25.3)$ & \\
weight (kg, mean, SE) & $5.3(0.32)$ & $4.9(0.26)$ & $0.33^{a}$ \\
\hline
\end{tabular}

Statistical analysis

Experimental data of tissue samples in the study are presented as box plot of the median and range of log-transformed relative expression level using Mann-Whitney $U$ test. While the data obtained from experiment in vitro assays are presented as mean \pm SEM from three separate experiments in triplicates per experiment by double-sided Student's t-test. Statistical analysis was performed by STATA 9.2 (Stata Corp, Texas, USA) and presented with Graph PAD prism software. Results were considered statistically significant at $\mathrm{P}<0.05$.

\section{Results}

\section{Subject characteristics}

A total of 163 human colon tissue specimens, including 88 HSCR cases and 75 matched controls, were enrolled in this study. The clinical information, including age, gender (Male/ Female) and body weight, was obtained from participants both HSCR cases and matched controls. As shown in Table 1, there was no significant difference in the distribution of age, sex and body weight between HSCR cases and matched controls. The gender rate (male/ female) of HSCR cases and controls were $9 / 2$ and 56/19, respectively.

\section{Down-regulation of miR-200a in HSCR patients}

As miR-200a was another key member of miR-200a family, we used qRT-PCR to examine the expression level in HSCR samples and control samples. The expression levels of miR200a was significantly lower in HSCR cases than those in matched controls as shown in Fig. $1 \mathrm{~A}$, indicating miR-200a might be involved in the pathological development of HSCR disease.

Mir-200a inhibitor inhibited cell migration and cell proliferation without affecting cell apoptosis and cell cycle progression

To investigate the functional roles of miR -200a, we then examined the effect of miR200a on cell migration, cell proliferation, cell apoptosis and cell cycle. By transfecting the 293T and SH-SY5Y cell lines with miR-200a inhibitor, the suppressive affection was observed in cell migration and proliferation by using the transwell assay and CCK8 assay, respectively. Both the number of migrated cells and the proliferation rate were significantly lower in the cells treated with miR-200a inhibitor, suggesting that inhibition of miR-200a suppress cell migration and cell proliferation (Fig. 2A, B). Additionally, we conducted the experiment using flow cytometry analysis to investigate whether miR-200a is involved in the apoptosis and cell cycle progression. However, the percentage of apoptosis cells was not statistically different between cells transfected with miR-200a inhibitor and the control while miR-200a inhibitor also did not affect cell distribution in cell cycle. (Fig. 2C, D).

Target prediction of miR-200a and miR-141

To predict potential common target genes that mediate function of migration and proliferation of miR-200a and miR-141, we utilized several target-prediction algorithms: miRBase, PicTar and TargetScan. Remarkably, PTEN and CXCL12, two proteins with 


\section{Cellular Physiology \\ and Biochemistry}

Cell Physiol Biochem 2014;34:543-553

\begin{tabular}{l|l}
\hline DOI: $10.1159 / 000363021$ & (C) 2014 S. Karger AG, Basel
\end{tabular}

\begin{tabular}{l|l} 
Published onlıne: August 08, 2014 & www.karger.com/cpb
\end{tabular}

Li et al.: MiR-200a/141 in Hirschsprung's Disease
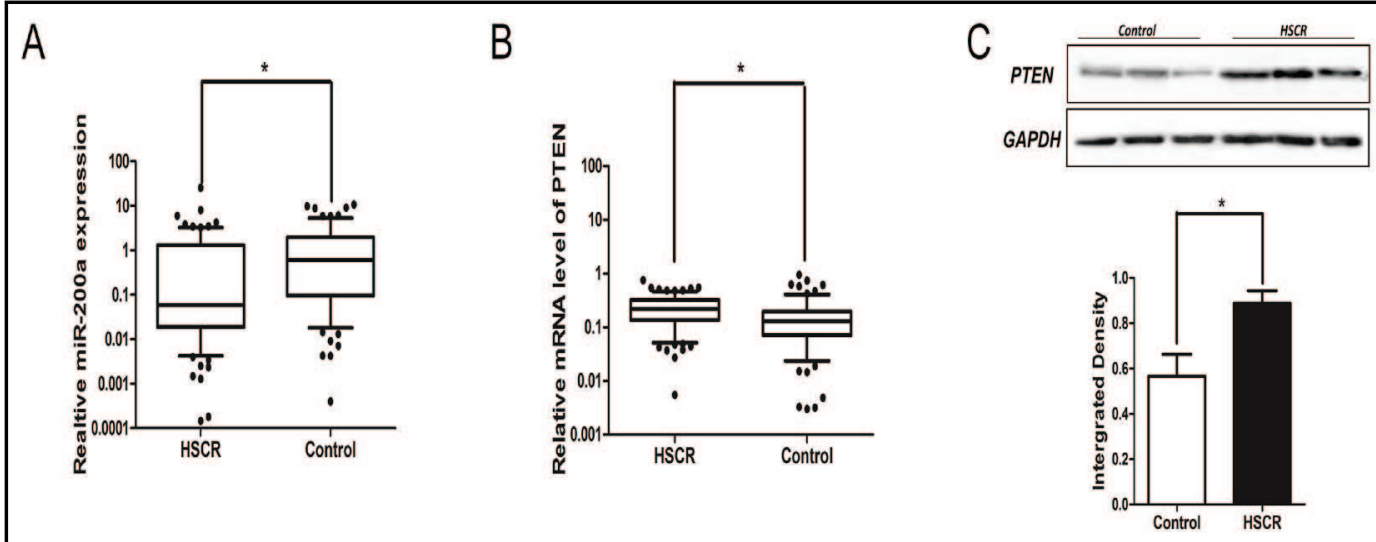

D

293T cell line

$E$

SH-SY5Y cell line
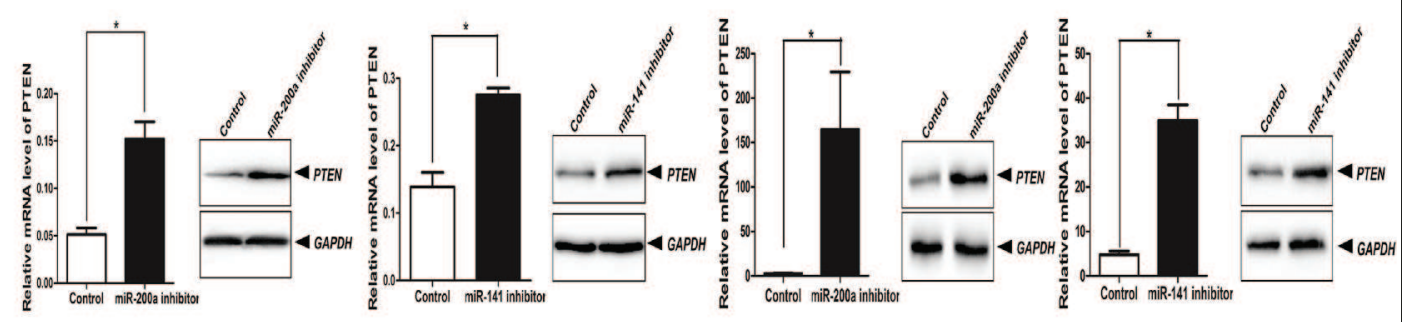

Fig. 1. MiR-200a was down-regulated while PTEN was up-regulated in HSCR patients and cell lines. A: The relative expression levels of miR-200a in human HSCR tissues $(n=88)$ and control tissues $(n=75)$ were evaluated by qRT-PCR. Data were presented as box plot of the median and range of log-transformed relative expression levels. The top and bottom of the box represent the seventy-fifth and twenty-fifth percentile. The whiskers indicate the 10 th and 90 th points. * Significantly different compared with that of control $(\mathrm{P}<0.05)$. B: The mRNA expression levels of PTEN in human HSCR tissues and control tissues. C: The protein expression levels of PTEN in human HSCR tissues and controls (3 representative samples from both groups are shown) (above). Quantization of Western-blotting was done by Image J software(blow). D-E: Cells were transfected with $100 \mathrm{nM}$ miR-200a inhibitor and miR-141 inhibitor for 48h, qRT-PCR was performed to evaluate the mRNA level of PTEN. PTEN protein expression levels were analyzed by western-blotting.

established functions of nerve cell migration and proliferation, are highly ranked in the results of predicted targets for miR-200a and miR-141.

\section{PTEN was up-regulated in HSCR tissues}

Samples of 88 HSCR cases and 75 matched controls were analyzed by the qRT-PCR for the mRNA expression of the predicted genes. PTEN mRNA expression level was significantly up-regulated in HSCR patients compared with normal controls (Fig. 1B). However, the expression levels of CXCL12 showed no differences between HSCR patients and matched controls. We further tested the protein levels of PTEN through the Western-blot, the result was consistent with its mRNA expression levels (Fig. 1C).

PTEN was the common target of miR-200a and miR-141

Two independent approaches were used to validate the miRNA-target interactions. The first was based on the secondary changes in mRNA and protein levels of the predicted gene after transfecting miR-200a/ miR-141 inhibitor and normal control. The second was to measure the effect of the miR-200a/ miR-141 inhibitor by detecting luciferase signal of a vector containing the 3'UTR of the predicted target. 


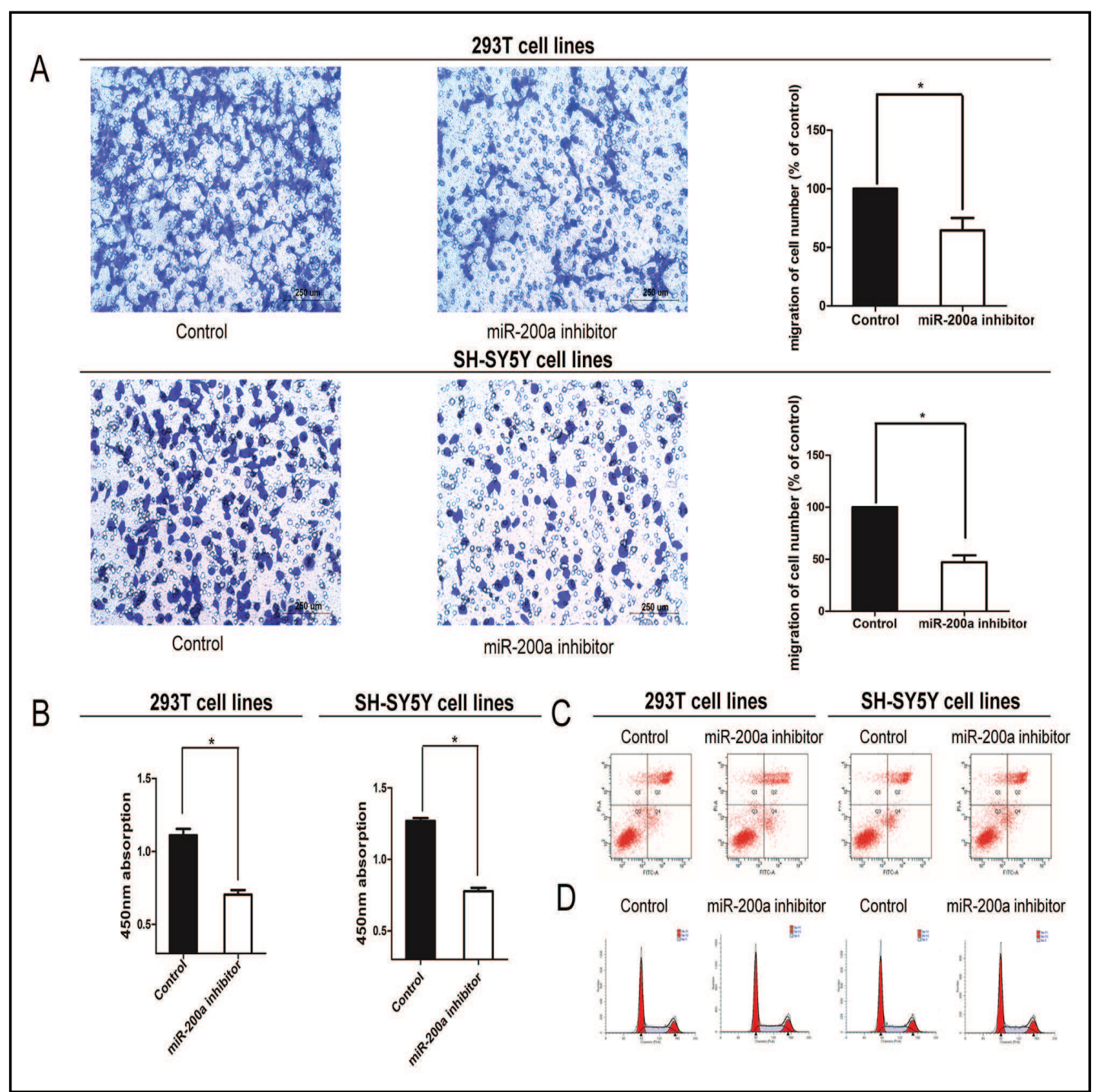

Fig. 2. Cytobiology change after treating cell lines with miR-200a inhibitor. A: Transwell assay was performed as described in Materials and Methods. The representative images of invasive cells at the bottom of the membrane stained with crystal violet were visualized as shown (left). The quantifications of cell migration were presented as percentage migrated cell numbers (right). * indicates significant difference compared with control group $(P<0.05)$. B: Absorbance at $450 \mathrm{~nm}$ was presented with Mean $\pm \mathrm{SE}$. ${ }^{*}$ indicates significant difference compared with control group $\mathrm{P}<0.05$. C-D: Flow cytometry assay was performed to assess cell apoptosis and cell cycle.

In the first approach, we transfected miR-200a/miR-141 inhibitor and normal control in $293 \mathrm{~T}$ and SH-SY5Y cell lines. PTEN mRNA levels were evaluated after $48 \mathrm{~h}$ transfection. As expected, PTEN expression was significantly increased at both the mRNA level and protein level in two cell lines (Fig. 1D, E).

In the second approach, a dual luciferase reporter system, which provides more evidence about a direct interaction between the miRNA and its target, was employed. The binding region of the 3'UTR of PTEN mRNA with wild type or mutant seed sequence recognizing sites was cloned to a Dual-Luciferase reporter (Fig. 4C). These constructs were referred to as pGL3-PTEN and pGL3-PTEN-mut. Co-transfection of miR-200a/miR-141 mimics with the pGL3-constructs to 293T and SH-SY5Y cell lines significantly suppressed luciferase activity noted only with the wild-type 3'-UTRs. The suppressive effect of miR-200a/miR-141 mimics 


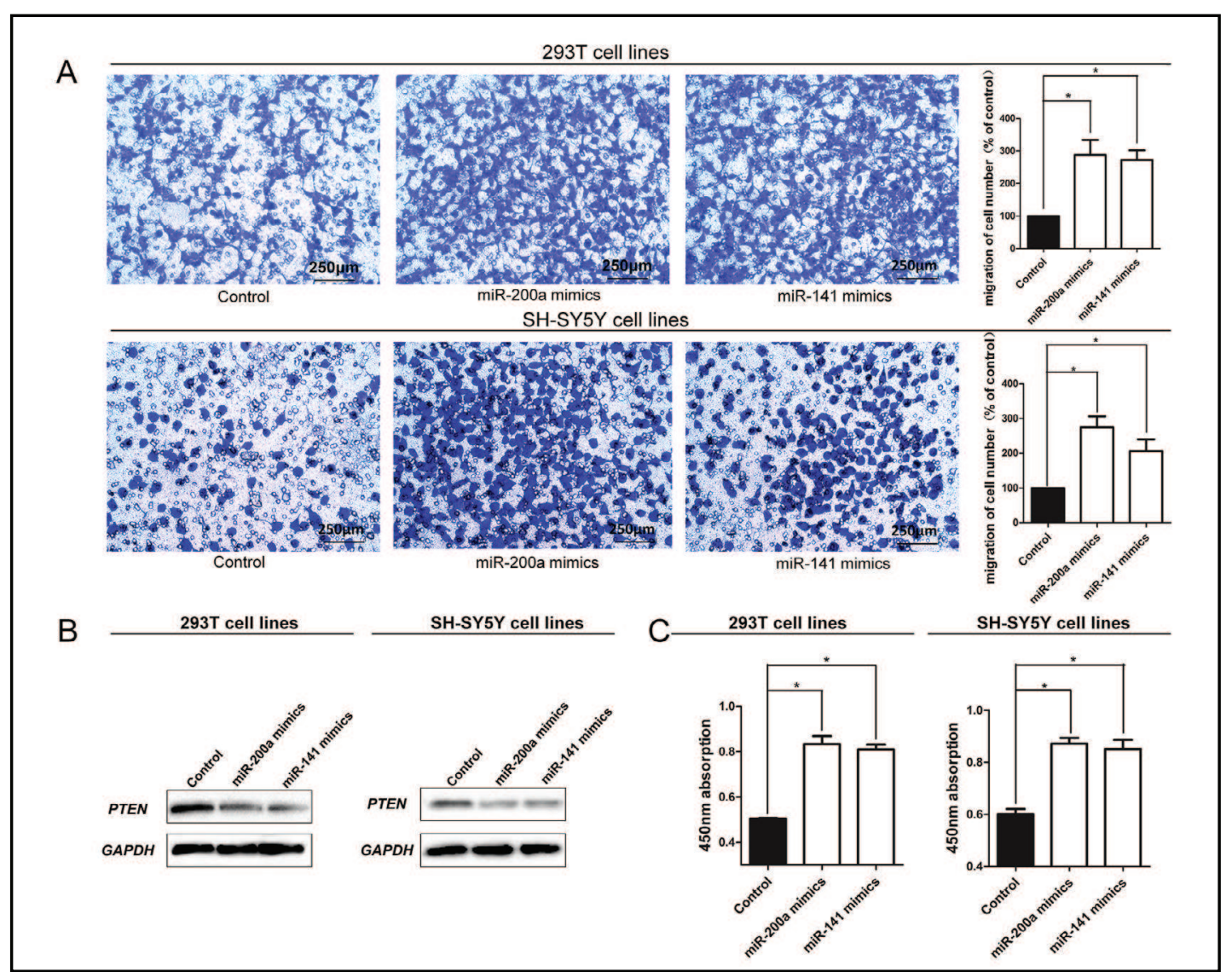

Fig. 3. Overexpression of miR-200a and miR-141 result in promotion of cell migration and proliferation, reduce the protein levels of the PTEN. A: The ability of the cells' migration were promoted after transfected with the miR-200a mimics and miR-141 mimics which were detected by transwell assay. The representative images of invasive cells at the bottom of the membrane stained with crystal violet were visualized as shown (left). The quantifications of cell migration were presented as percentage migrated cell numbers (right). B: Cells were transfected with $50 \mathrm{nM}$ miR-200a mimics and miR-141 mimics for 48h, the downregulation of PTEN protein expression levels were detected by western-blotting assay. C: Assessment of cell proliferation by CCK8 assay. Absorbance at $450 \mathrm{~nm}$ was presented with Mean \pm SE. All tests were performed in triplicate, * indicates significant difference compared with control group $\mathrm{P}<0.05$.

was abrogated when pGL3-PTEN-mut was applied; confirming PTEN was the directly target of miR-200a and miR-141(Fig. 4D).

Overexpression of miR-200a and miR-141 result in promotion of cell migration and proliferation, reduce the protein levels of the PTEN.

To explore the role of miR-200 family involved in HSCR, we transfected 293T and SHSY5Y cell lines with miR-200a and miR-141 mimics to upregulate their expression. The transwell assay and CCK8 assay showed that the migration and proliferation rate of 293T and SH-SY5Y cells were significant increased after overexpression of miR-200a and miR-141 (Fig. 3A, C). The downregulation of the PTEN protein levels were also detected by western blot assay after the transfection ( Fig. 3B).

PTEN SiRNA partially rescued miR-200a/miR-141 inhibitor-mediated cell migration and cell proliferation.

To investigate whether the decreased level of PTEN is directly contributing to the suppressed cell migration and cell proliferation induced by the miR-200a/miR-141 inhibitor, 


\section{Cellular Physiology and Biochemistry}

Cell Physiol Biochem 2014;34:543-553

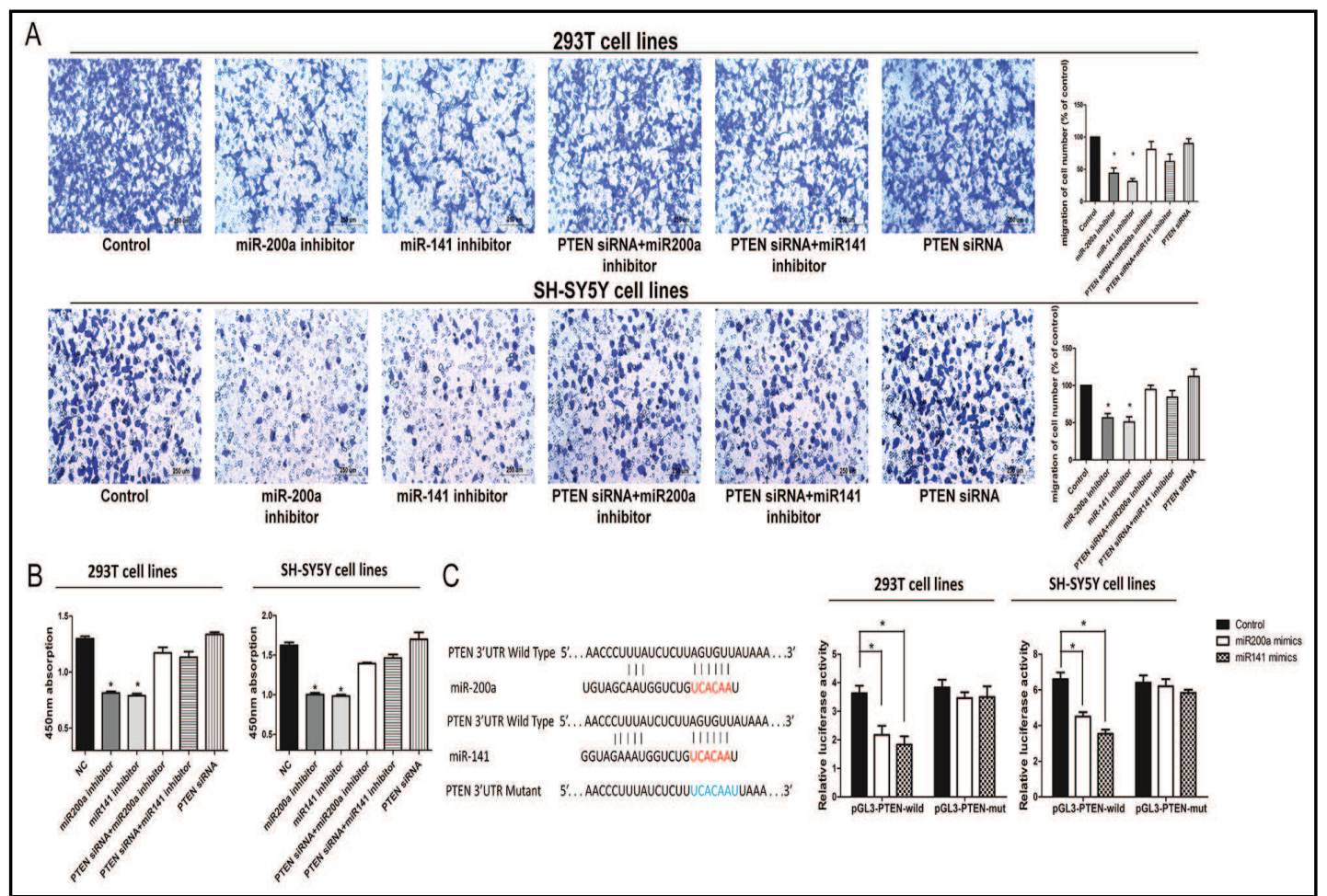

Fig. 4. Migration and proliferation were reversed after co-transfected with miR-200a inhibitor, miR-141 inhibitor and PTEN siRNA. A-B: The cell biology changes in migration and proliferation were reversed after co-transfected miR-200a inhibitor, miR-141 inhibitor and PTEN siRNA. Data were presented as mean \pm SEM from three separate experiments performed in triplicates, and were analyzed by double-sided Student's t-test. ( ${ }^{*}$ indicates $P<0.05$ ). C: Sequence alignment of human miR-200a and miR-141 with 3' UTR of PTEN. Bottom: mutations in the $3^{\prime}$-UTR of PTEN in order to create the mutant luciferase reporter construct. D: Cells were co-transfected with miR-200a and miR-141 mimics or miR-control, renilla luciferase vector pRLSV40 and PTEN 3'UTR luciferase reporters for $48 \mathrm{~h}$. Both firefly and Renilla luciferase activities are measured in the same sample. Firefly luciferase signals were normalized with Renilla luciferase signals. All tests were performed in triplicate and presented as mean \pm SE.

a set of rescue experiments were conducted. We co-transfection miR-200a/miR-141 inhibitor and specific small interference RNAs (siRNAs) designed to knock-down PTEN in 293T and SH-SY5Y cell lines. As presented in Fig. 4A, knock-down of PTEN by the individual cognate siRNAs significantly attenuated the suppression in migration when compared with miR-200a/miR-141 inhibition group, but still weaker when compared to the control. Cell migration ability transfected with PTEN siRNAs exhibited normal migration when compared to the control. While decreasing the level of PTEN rescued the miR-200a/miR-141-induced cell migration. Consistent with the results obtained with the effects on cell migration, we also found the same results in the CCK8 assay when co-transfection miR-200a/miR-141 inhibitor and PTEN siRNAs in 293T and SH-SY5Y cell line (Fig. 4B). These data indicated that significant reduction of miR-200a/miR-141 caused the impairment of cells proliferation and migration by keeping the high levels of PTEN.

\section{Discussion}

Aberrant expression of several microRNAs, accounts for profound pathological effects associated with disease initiation and/or progression, is closely associated with human disorder. For instance, the miR-17-92 cluster and its paralogs at the nexus of critical pathways 


\section{Cellular Physiology and Biochemistry}

Cell Physiol Biochem 2014;34:543-553

\begin{tabular}{l|l}
\hline DOI: $10.1159 / 000363021$ & C) 2014 S. Karger AG, Basel
\end{tabular}

www.karger.com/cpb

Li et al.: MiR-200a/141 in Hirschsprung's Disease

regulate cellular life and death decisions during normal development and in malignancy [14]. Down regulation of miR-192, miR-194 and miR-215 suppressing tumor progression in renal cell carcinoma [15]. In our previous work, we identified miR-141, a member of the human miR-200 family, took effects on the pathogenesis of HSCR. The miR-200 family consists of paralogs located on two chromosomal regions, based on the seed region (nucleotides 2-7), they can be divided into two sequence clusters: miR-200b, miR-200c and miR-429, as well as miR-200a and miR-141. It is known that the miR-200 family plays a significant role in growth, invasion and migration [16]. Several studies showed that the family regulate epithelial to mesenchymal transition, the initiating step of metastasis, by targeting ZEB1 and SIP1 [17] and was involved in olfactory neurogenesis [9]. To date, functions of miR-200 family deregulated in HSCR have not been fully characterized. In this study, we demonstrated for the first time that the down-regulation of miR-200a and miR-141, a cluster of the miR200 family, participanted in cell migration and proliferation by directly targeting PTEN in HSCR.

A controversial role of the miR-200a and miR-141 has already been discussed in other disease. MiR-141 and miR-200a are involved in pre-osteoblast differentiation in part by regulating the expression of Dlx5 [18] and act on ovarian tumorigenesis by controlling oxidative stress response [19]. In the current study, we demonstrated that the expression of miR-200a was markedly down-regulated in HSCR tissues in accordance with the miR-141. Functionally, suppressive expression of miRNA-200a and miR-141 in cultured human 293T and SH-SY5Y cells inhibited cell migration and proliferation. To grasp full comprehension of how miR-200a and miR-141 mediates the function of these cells, PTEN has been identified as the common targets for miR-200a and miR-141 using several different miRNA targetprediction programs and it turned out that expression of PTEN was significantly increased in HSCR samples, compared to the matched control tissue. It is proven that PTEN mediates many of its effects on proliferation, growth, survival and migration through suppressing phosphoinositide 3-kinase (PI3K)-dependent signaling pathways [20], acting as a tumor suppressor gene implicated in a wide variety of human cancers [21]. However, deletion of PTEN in mice reveals that it is essential for development [22]. Immunohistochemical analyses in humans showed that PTEN is abundant in the nervous plexus of the gastrointestinal system during fetal development [23]. Furthermore, the crosstalk between PTEN and RET signaling pathways which have been identified to be associated with the pathogenesis of HSCR [24]. Due to the profound effects of PTEN on the function of cell growth, proliferation in the development and physiology, we assumed that PTEN played a crucial role during ENS development in fetal period. So we speculated that reduced miR-200a and miR-141 by up regulating PTEN plays a certain role in neural crest cell proliferation, migration, which may contribute to HSCR initiation. Firstly, the down-regulation of PTEN was confirmed in HSCR tissue and subsequently, the changes in mRNA and protein levels of PTEN were detected after the transfection with miR-200a or miR-141 inhibitor. Combining with the target reporter assays, we further have demonstrated that miR-200a and miR-141 post-transcriptionally regulates PTEN via binding the 3'-UTR of PTEN mRNA. Moreover, contrary to inhibition of miRNA-200a and miR-141 in cultured human 293T and SH-SY5Y cells, overexpression of miR-200a and miR-141 resulted in promotion of cell migration and proliferation, with the the downregulation of the PTEN protein levels, were in accordance with other studies that inhibition of PTEN was responsible for the increased proliferation. The results also demonstrated that PTEN was involved in the inhibitory function of miR-200 family in HSCR. Finally a set of the rescue experiments in vitro were performed to confirm that the miR200a/miR-141/PTEN signaling pathway contributes to pathogenic mechanism of HSCR disease.

Taken together, the current study revealed a novel mechanism in the pathogenesis of HSCR. The miR-200a/miR-141/PTEN signaling pathway was associated with early migration and proliferation in HSCR tissue and related cell lines, which might contribute to the development of HSCR disease. In addition, more deep researches are needed to validate our findings and investigate the molecular mechanisms of HSCR in the future. 


\section{Cellular Physiology and Biochemistry}

Cell Physiol Biochem 2014;34:543-553

\begin{tabular}{l|l}
\hline DOI: 10.1159/000363021 & (c) 2014 S. Karger AG, Basel
\end{tabular}

\begin{tabular}{l|l} 
Published online: August 08, 2014 & www.karger.com/cpb
\end{tabular}

Li et al.: MiR-200a/141 in Hirschsprung's Disease

\section{Acknowledgements}

We thank Dr. Jie Zhang, HuanChen and Changgui Lu (Nanjing Children's Hospital Affiliated to Nanjing Medical University) for sample collection.

This study was supported by Natural Science Foundation of China [NSFC 81370473], Natural Science Foundation of Jiangsu Province of China [BK20131388] and The Scientific Research Project of Jiangsu Provincial Department of health [H201342] and Priority Academic Program Development of Jiangsu Higher Education Institutions (PAPD).

\section{Disclosure Statement}

There are no conflicts of interest.

\section{References}

1 Gariepy CE: Intestinal motility disorders and development of the enteric nervous system. Pediatr Res 2001;49:605-613.

2 Spouge D, Baird P: Hirschsprung disease in a large birth cohort. Teratology 1985;32:171-177.

-3 Carter TC, Kay DM, Browne ML, Liu A, Romitti PA, Kuehn D, Conley MR, Caggana M, Druschel CM, Brody LC: Hirschsprung's disease and variants in genes that regulate enteric neural crest cell proliferation, migration and differentiation. J Hum Genet 2012;57:485-493.

4 Badner JA, Sieber W, Garver K, Chakravarti A: A genetic study of hirschsprung disease. Am J Hum Genet 1990;46:568.

5 Butler Tjaden NE, Trainor PA: The developmental etiology and pathogenesis of hirschsprung disease. Transl Res 2013;162:1-15.

6 Bartel DP: Micrornas: Genomics, biogenesis, mechanism, and function. cell 2004;116:281-297.

7 Senanayake U, Das S, Vesely P, Alzoughbi W, Fröhlich LF, Chowdhury P, Leuschner I, Hoefler G, Guertl B: Mir-192, mir-194, mir-215, mir-200c and mir-141 are downregulated and their common target acvr2b is strongly expressed in renal childhood neoplasms. Carcinogenesis 2012;33:1014-1021.

-8 Leskelä S, Leandro-García LJ, Mendiola M, Barriuso J, Inglada-Pérez L, Muñoz I, Martínez-Delgado B, Redondo A, de Santiago J, Robledo M: The mir-200 family controls $\beta$-tubulin iii expression and is associated with paclitaxel-based treatment response and progression-free survival in ovarian cancer patients. Endocr Relat Cancer 2011;18:85-95.

-9 Choi PS, Zakhary L, Choi W-Y, Caron S, Alvarez-Saavedra E, Miska EA, McManus M, Harfe B, Giraldez AJ, Horvitz RH: Members of the mirna-200 family regulate olfactory neurogenesis. Neuron 2008;57:41-55.

-10 Uhlmann S, Zhang J, Schwäger A, Mannsperger H, Riazalhosseini Y, Burmester S, Ward A, Korf U, Wiemann S, Sahin Ö: Mir-200bc/429 cluster targets plc 1 and differentially regulates proliferation and egf-driven invasion than mir-200a/141 in breast cancer. Oncogene 2010;29:4297-4306.

11 Tang W, Qin J, Tang J, Zhang H, Zhou Z, Li B, Geng Q, Wu W, Xia Y, Xu X: Aberrant reduction of mir-141 increased cd47/cul3 in hirschsprung's disease. Cell Physiol Biochem 2013;32:1655-1667.

-12 Korpal M, Lee ES, Hu G, Kang Y: The mir-200 family inhibits epithelial-mesenchymal transition and cancer cell migration by direct targeting of e-cadherin transcriptional repressors zeb1 and zeb2. J Biol Chem 2008;283:14910-14914.

13 Martelli A, Evangelisti C, Chappell W, Abrams S, Bäsecke J, Stivala F, Donia M, Fagone P, Nicoletti F, Libra M: Targeting the translational apparatus to improve leukemia therapy: Roles of the pi3k/pten/akt/mtor pathway. Leukemia 2011;25:1064-1079.

14 Mendell JT: Miriad roles for the mir-17-92 cluster in development and disease. Cell 2008;133:217-222.

15 Khella HW, Bakhet M, Allo G, Jewett MA, Girgis AH, Latif A, Girgis H, Von Both I, Bjarnason GA, Yousef GM: Mir-192, mir-194 and mir-215: A convergent mirna network suppressing tumor progression in renal cell carcinoma. Carcinogenesis 2013;34:2231-2239. 
16 Roybal JD, Zang Y, Ahn Y-H, Yang Y, Gibbons DL, Baird BN, Alvarez C, Thilaganathan N, Liu DD, Saintigny P: Mir-200 inhibits lung adenocarcinoma cell invasion and metastasis by targeting flt1/vegfr1. Mol Cancer Res 2011;9:25-35.

17 Gregory PA, Bert AG, Paterson EL, Barry SC, Tsykin A, Farshid G, Vadas MA, Khew-Goodall Y, Goodall GJ: The mir-200 family and mir-205 regulate epithelial to mesenchymal transition by targeting zeb1 and sip1. Nat Cell Biol 2008;10:593-601.

-18 Itoh T, Nozawa Y, Akao Y: Microrna-141 and-200a are involved in bone morphogenetic protein-2-induced mouse pre-osteoblast differentiation by targeting distal-less homeobox 5. J Biol Chem 2009;284:1927219279.

19 Mateescu B, Batista L, Cardon M, Gruosso T, de Feraudy Y, Mariani O, Nicolas A, Meyniel J-P, Cottu P, SastreGarau X: Mir-141 and mir-200a act on ovarian tumorigenesis by controlling oxidative stress response. Nat Med 2011;17:1627-1635.

20 Leslie NR, Yang X, Downes CP, Weijer CJ: Ptdins $(3,4,5) \mathrm{p}<$ sub $>3</$ sub $>$-dependent and-independent roles for pten in the control of cell migration. Curr Biol 2007;17:115-125.

21 Tamura M, Gu J, Matsumoto K, Aota S-i, Parsons R, Yamada KM: Inhibition of cell migration, spreading, and focal adhesions by tumor suppressor pten. Science 1998;280:1614-1617.

22 Cristofano AD, Pesce B, Cordon-Cardo C, Pandolfi PP: Pten is essential for embryonic development and tumour suppression. Nat Genet 1998;19:348-355.

23 Gimm 0, Attié-Bitach T, Lees JA, Vekemans M, Eng C: Expression of the pten tumour suppressor protein during human development. Hum Mol Genet 2000;9:1633-1639.

24 Zbuk KM, Eng C: Cancer phenomics: Ret and pten as illustrative models. Nat Rev Cancer 2006;7:35-45. 